\title{
NASA Integrated Space Communications Network
}

\author{
Wallace Tai ${ }^{1}$ \\ NASA Caltech/Jet Propulsion Laboratory, Pasadena, California, 91109, USA \\ Nate Wright ${ }^{2}$ and Mike Prior ${ }^{3}$ \\ NASA Goddard Space Flight Center, Greenbelt, Maryland, 20771, USA \\ and \\ Kul Bhasin ${ }^{4}$ \\ NASA Glenn Research Center, Cleveland, Ohio, 44135, USA
}

\begin{abstract}
The NASA Integrated Network for Space Communications and Navigation (SCaN) has been in the definition phase since 2010. It is intended to integrate NASA's three existing network elements, i.e., the Space Network, Near Earth Network, and Deep Space Network, into a single network. In addition to the technical merits, the primary purpose of the Integrated Network is to achieve a level of operating cost efficiency significantly higher than it is today. Salient features of the Integrated Network include (a) a central system element that performs service management functions and user mission interfaces for service requests; (b) a set of common service execution equipment deployed at the all stations that provides return, forward, and radiometric data processing and delivery capabilities; (c) the network monitor and control operations for the entire integrated network are conducted remotely and centrally at a prime-shift site and rotating among three sites globally (a followthe-sun approach); (d) the common network monitor and control software deployed at all three network elements that supports the follow-the-sun operations.
\end{abstract}

\section{Introduction}

THE NASA Integrated Space Communications Network is intended to unify NASA's three existing network elements, i.e., the Space Network, Near Earth Network, and Deep Space Network, into a single network. This paper describes the architecture of the integrated network concluded so far by the NASA-wide study. ${ }^{1,2}$ It focuses on the integration of the diverse, heterogeneous communications assets and their operations, but does not cover the technology advancement aspect of space communications, e.g., optical communications, at the network. Figure 1 illustrates the "should-be" architecture to be realized around the 2018 era.

In this context, being "integrated" (adj.) means "making the elements of a system function coherently". Clearly, the "system" here is the NASA integrated network and the "elements" are those equivalents of present Space Network (SN), Near Earth Network (NEN), and Deep Space Network (DSN). To avoid any confusion by the names of these networks, the elements or network elements of the system, i.e., the integrated network, are now changed to Earth Based Relay Element (EBRE), Near Earth Element (NEE), and Deep Space Element (DSE). The architecture options considered for this study include three approaches: asset-specific (retaining the current unique equipment of all three network elements), common (migrating to a single design that is deployed to the three network elements with some network element-specific adaptation), and centralized (migrating to a single design that is implemented

${ }^{1}$ Chief Engineer, NASA/JPL Interplanetary Network Directorate, Mail Stop 126-200, 4800 Oak Grove Drive, Pasadena, CA 91109, USA

2 Network Systems Integration Manager, NASA/GFSC Exploration and Space Communications Projects Division/Code 450, 8800 Greenbelt Road Greenbelt, MD 20771, USA

${ }^{3}$ Network Systems Engineer, ARES Corporation, NASA/GFSC Exploration and Space Communications Projects Division/Code 450, 8800 Greenbelt Road Greenbelt, Maryland 20771, USA

${ }^{4}$ Project Manager, Program System Engineering, NASA/GRC, 21000 Brookpark Road, Cleveland, OH 44135, USA 
once to unified operation of the combined networks). The following matrix shows five possibilities, i.e., combinations of various implementation, deployment, and operations approaches, in selecting architecture options:

\begin{tabular}{|l|c|c|c|c|}
\hline & $\begin{array}{l}\text { Centrally deployed, } \\
\text { centrally operated }\end{array}$ & $\begin{array}{l}\text { Multiply deployed, } \\
\text { multiply operated }\end{array}$ & $\begin{array}{l}\text { Centrally deployed, } \\
\text { multiply operated }\end{array}$ & $\begin{array}{l}\text { Multiply deployed, } \\
\text { centrally operated }\end{array}$ \\
\hline Singly implemented & $\mathrm{X}$ & $\mathrm{X}$ & $\mathrm{X}$ & $\mathrm{X}$ \\
\hline $\begin{array}{l}\text { Commonly, multiply } \\
\text { implemented }\end{array}$ & $\mathrm{N} / \mathrm{A}$ & $\mathrm{X}$ & $\mathrm{N} / \mathrm{A}$ & N/A \\
\hline
\end{tabular}

Since all three individual network elements can functionally be decomposed into three top-level functions: service execution, service management, and network control, we can address the integrated network in terms of integrating each of the three functions across the three network elements. As such, architecture options are identified for the integrated service execution (ISE), integrated service management (ISM), and integrated network control (INC) functions, respectively. Furthermore, for purpose of allocating functional capabilities to physical system entities, we have identified three types of physical system entities, i.e., the ground station site (GSS), the Network Operations Node (NON), and the Integrated Network Operations Center (INOC), for the integrated network. The integrated network architecture in terms of the three functions is described in Section IV of this paper.

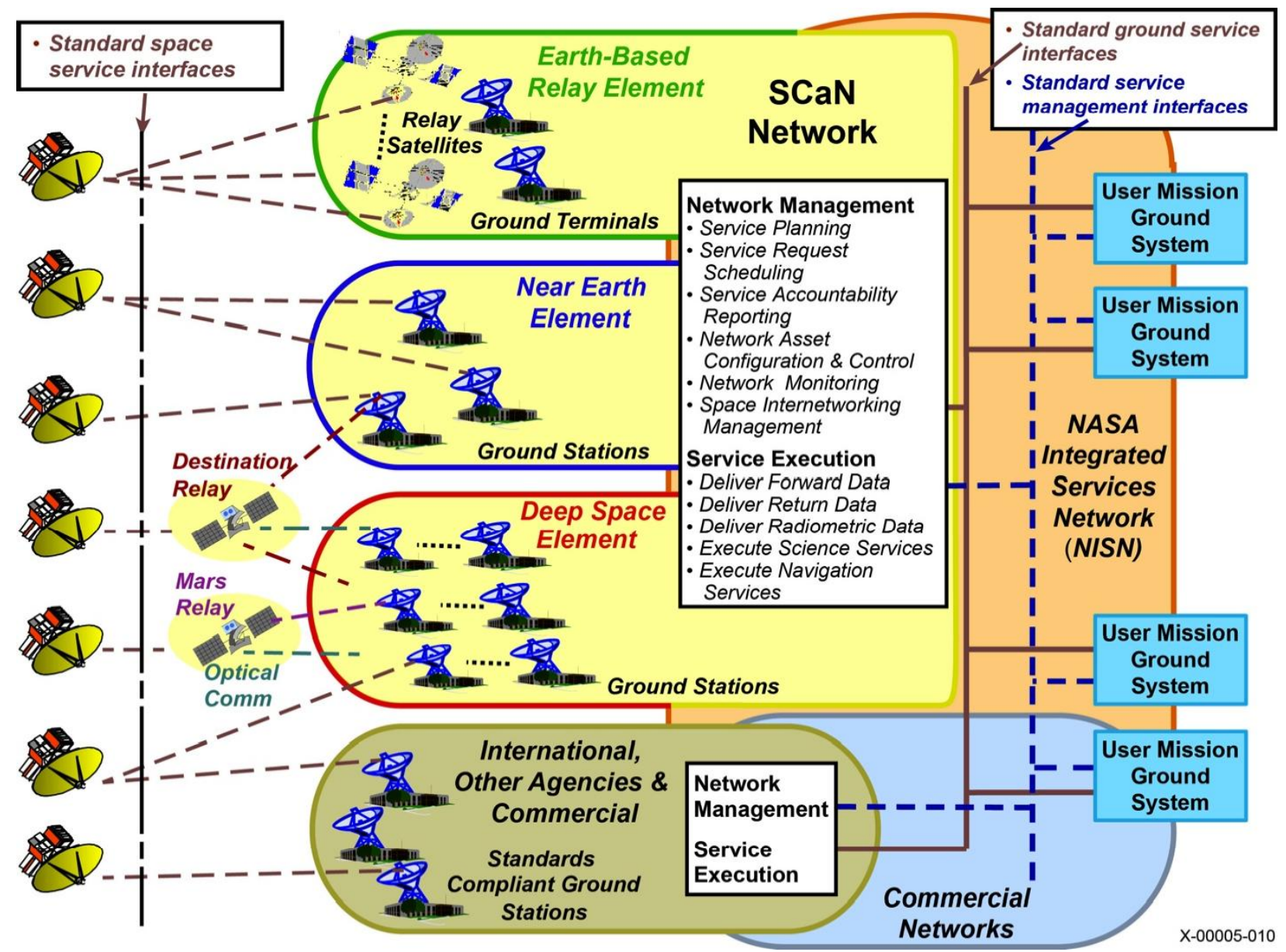

Figure 1. The NASA Integrated Network Architecture around 2018 era - A Notional Illustration

\section{Key Characteristics of NASA's Present Space Communications Network}

Key characteristics of the present space communications network(s) can be summarized are as follows:

1) Diverse, heterogeneous communications assets: NASA currently operates complex space and ground infrastructure that supports all mission domains. The SN consists of a constellation of geosynchronous 
relays to support missions in equatorial to highly inclined orbits from launch to Low Earth Orbit (LEO). The NEN consists of a set of NASA-owned and commercial ground stations for missions operating in orbital and suborbital locations, including LEO, GEO, and highly elliptical orbits and at lunar distances. The DSN consists of a set of large-aperture ground stations providing coverage of satellites from aboveGEO distance to the edge of our solar system. The present network is very capable, but is also complex because of the heterogeneous nature of the component network assets and the lack of consistent service offerings, interfaces, and interoperability.

2) No explicit, formal integrated network architecture: The NASA space communications infrastructure evolved into three distinct, unrelated component networks that provide domain-specific communications services. Until now, there has been no explicit network architecture documented using a formal methodology, and agreed by the Agency, to provide guidance to the evolution of an integrated space communications infrastructure.

3) Independently evolved and operated networks: The present NASA space communication network elements have been independently evolving for as long as four decades on their own respective paths to provide TT\&C services to flight missions. Infusion of new technologies and capabilities into each network has followed a road map at the individual network level. Each network is a distinct service-providing system largely functioning as a self-contained element. This approach offers certain strengths in that it allows each network to focus on the operational capabilities needed by its primary mission customer base using the most cost-effective and lowest risk approach possible. However, the present architecture does not include an orchestrated evolutionary path encompassing all networks.

4) Mission requirements-driven network architecture: The evolution of the various networks has largely been driven by mission requirements. The growth in network capabilities very often depends on the needs of the few NASA flagship missions that have sufficient funding flexibility to advocate for more advanced performance and functionality. This factor contributes to the networks different services and to a slow, inefficient process for infusing new communications technology into the operational network assets.

5) Diversely distinct service paradigms among the networks: While all network elements provide TT\&C functionality, the types and levels of services offered by the SN, NEN, and DSN are very different. At the overall NASA service architecture level, there are few common, standard services defined for all three networks, and there is no uniform approach to establish standard service interfaces between the networks and customer missions. This network element-specific service provision approach has a number of deficiencies, particularly for mission users who need services from more than one network, and it impacts users during development, testing, and operations phases. Moreover, each network element employs its own tools and processes for service management. As a result, they offer user missions different degrees of controllability and visibility into network assets. The network-specific service management approach has served customers fairly well for a long time; but, as NASA moves into the next decade of space exploration, the Agency must adopt a more efficient, cost-effective approach to managing space communication services. A more integrated approach to managing services is urgently needed.

6) Varying degree of interoperability within NASA and with networks of non-NASA space agencies: When providing support to missions requiring services of more than one NASA network element or the networks of non-NASA space agencies, the three NASA network elements take different approaches to accomplish interoperability. In some cases, the approach taken by a network is ad hoc, varies from mission to mission, and depends on the particular assets involved in the cross support.

7) Tool-assisted, operator-tended service providing systems: A common trait shared by the three NASA network elements is that they all rely heavily on human-operated tools for conducting day-to-day service provision activities. These activities include network scheduling, configuration set-up, monitoring and controlling the assets during the pass, and anomaly detection and recovery. The network elements differ, however, in the degree of automation applied. None of them has reached the state of unattended operations for nominal mission event support.

8) Network-specific service pricing, cost attribution and accounting: Primarily due to the separate management of the network elements and their heterogeneous assets, NASA established different policies governing the pricing, cost attribution, and accounting of the services provided by the three networks. Service pricing algorithms, e.g., aperture fee calculations are network-dependent. Rules governing attribution of cost to user missions vs. the network elements are also different. 


\section{Key Characteristics of the NASA Integrated Space Communications Network}

The NASA integrated network that encompasses the three existing heterogeneous networks (hereafter labeled as "network elements") must exhibit two important behaviors: (a) the integrated network as a whole or in part through each network element "behaves" as a single service providing system to user missions; (b) within the integrated network its hardware/software components and operational processes across the three network elements share a high degree of commonality and coherence. As shown in Figure 1, to the user missions the integrated network possesses the following key characteristics:

- Provision of internationally interoperable, standard services to user missions by all network elements

- Provision of standard service interfaces between all network assets and the ground systems of user missions

- Provision of standard interfaces between all network assets and the flight systems of user missions over the space link.

- Provision of standard service management capabilities for user missions to subscribe and request the space communications and tracking services.

Key attributes including those associated with commonality and coherence internal to the integrated network are itemized in the following table:

\begin{tabular}{|c|c|}
\hline Function & Key Attributes \\
\hline \multirow[t]{5}{*}{ Service Management } & Common (but distributed) or physically centralized service management (to be decided). \\
\hline & $\begin{array}{l}\text { A Service Portal functions as the primary access point for user missions to interface with } \\
\text { the NASA Integrated Network for service management capabilities. }\end{array}$ \\
\hline & Integrated service planning process and team $(\mathrm{s})$ \\
\hline & $\begin{array}{l}\text { Central scheduling user interface capabilities for user missions to schedule services } \\
\text { provided by all or any of the } 3 \text { network elements. }\end{array}$ \\
\hline & Scheduling engine: common or network element-specific (to be decided). \\
\hline \multirow[t]{6}{*}{ Network Control } & Fully integrated network control across all 3 network elements. \\
\hline & Common network control software deployed at all 3 network elements. \\
\hline & $\begin{array}{l}\text { Integrated network control operations: Operational activities for network control are } \\
\text { conducted globally at three Network Operations Nodes (NONs) where each of the three } \\
\text { NONs monitors and controls the entire integrated network (EBRE, NEE and DSE) during a } \\
\text { single prime-shift. A follow-the-sun, remote operations for network monitor \& control. }\end{array}$ \\
\hline & $\begin{array}{l}\text { Common network control software derived from EBRE elements with network element- } \\
\text { specific specialization and adaptation. }\end{array}$ \\
\hline & $\begin{array}{l}\text { Common, standard interfaces with MOCs, service management elements, service execution } \\
\text { elements, and network control operators. }\end{array}$ \\
\hline & $\begin{array}{l}\text { A Service Portal functions as the virtually centralized interface point, for network monitor } \\
\text { and control with user missions. }\end{array}$ \\
\hline \multirow[t]{4}{*}{ Service Execution } & Standard services and service interfaces provided at all 3 network elements. \\
\hline & $\begin{array}{l}\text { Common (but distributed) service execution capabilities for all } 3 \text { network elements. High- } \\
\text { degree of commonality in service execution equipment at all ground station sites (GSS) for } \\
\text { all network elements. }\end{array}$ \\
\hline & $\begin{array}{l}\text { Common service execution capabilities based on EBRE developed capabilities (hardware } \\
\& \text { software) with network asset-specific adaptation. }\end{array}$ \\
\hline & $\begin{array}{l}\text { A Service Portal functions as the virtually centralized data delivery point, i.e., the "service } \\
\text { dispatcher", to user missions. }\end{array}$ \\
\hline
\end{tabular}

\section{Integrated Space Communications Network - System Design}

This section addresses the integrated network architecture in terms of the integrated service execution (ISE), integrated service management (ISM), and integrated network control (INC) functions.

\section{A. Integrated Service Execution (ISE)}

The Service Execution function includes four types of services: the forward, return, and radiometric data processing and delivery services, plus position and timing service. For integrating these service execution functions across all three network elements, allocation of data processing and delivery capabilities at different levels, i.e., RF, 
bit stream, frame, packet, and file, to the various physical system elements, i.e., ground stations sites (GSSs) locally vs. the integrated network operations center (INOC) centrally. The following table describes the options assessed:

\begin{tabular}{|c|c|}
\hline Option & Description \\
\hline ISE-1 & Both processing ${ }^{1}$ and data delivery/ transfer at Ground Station Sites ${ }^{2}$ \\
\hline$\overline{\text { ISE-2 }}$ & $\begin{array}{l}\text { Processing }^{1} \text { at ground station sites } \\
\text { Center } \text {; data delivery/transfer at the Integrated Network Operations }\end{array}$ \\
\hline ISE-3 & Both processing ${ }^{1}$ and data delivery/ transfer at the $\mathrm{INOC}^{3}$ \\
\hline$\overline{\text { ISE-4 }}$ & Both processing ${ }^{4}$ and data delivery/ transfer at the $\mathrm{INOC}^{3}$ \\
\hline $\begin{array}{l}\text { Notes: } \\
\text { domains } \\
{ }^{1} \text { Proces } \\
\text { service } \\
\text { Statior } \\
2 \text { A GSS } \\
\text { infrast } \\
3 \text { The IN } \\
\text { and ne } \\
4 \text { Proces }\end{array}$ & $\begin{array}{l}\text { options are predicated upon the same degree of commonality in service capabilities among all mission } \\
\text { g., LEO, HEO, and deep space. } \\
\text { gg associated with TT\&C services is at the levels above bit stream level. That means for return link } \\
\text { regardless of which option (ISE-1 - ISE-3), at least the bit stream will be generated at the Ground } \\
\text { ites (GSS), and for command processing the GSS will always receive at least encoded bit stream. } \\
\text { as one or more ground antennas with associated TT\&C processing equipment with shared } \\
\text { cture, e.g. power, HVAC, frequency and timing, ground communications, and buildings. } \\
\text { C is a physical system element that performs the operations associated with the service management } \\
\text { ork control functions for all the communications assets and elements. } \\
\text { ig associated with TT\&C services is at the levels including and above bit stream level. }\end{array}$ \\
\hline
\end{tabular}

After evaluating all options against a set of figures of merit (FOMs) and taking into account the risks, and cost estimates, the ISE-1, i.e., both data processing ${ }^{1}$ and delivery at the individual ground station sites, is selected as the best-value alternative.

Figure 2 depicts the integrated service execution architecture. Each of the network elements continues to provide its domain specific processing, and each ground station site does all of the forward and return data processing. Common data delivery services and interfaces are provided (as shown in the same color codes for network elements). Figure 3 shows the physical topology of the same architecture.

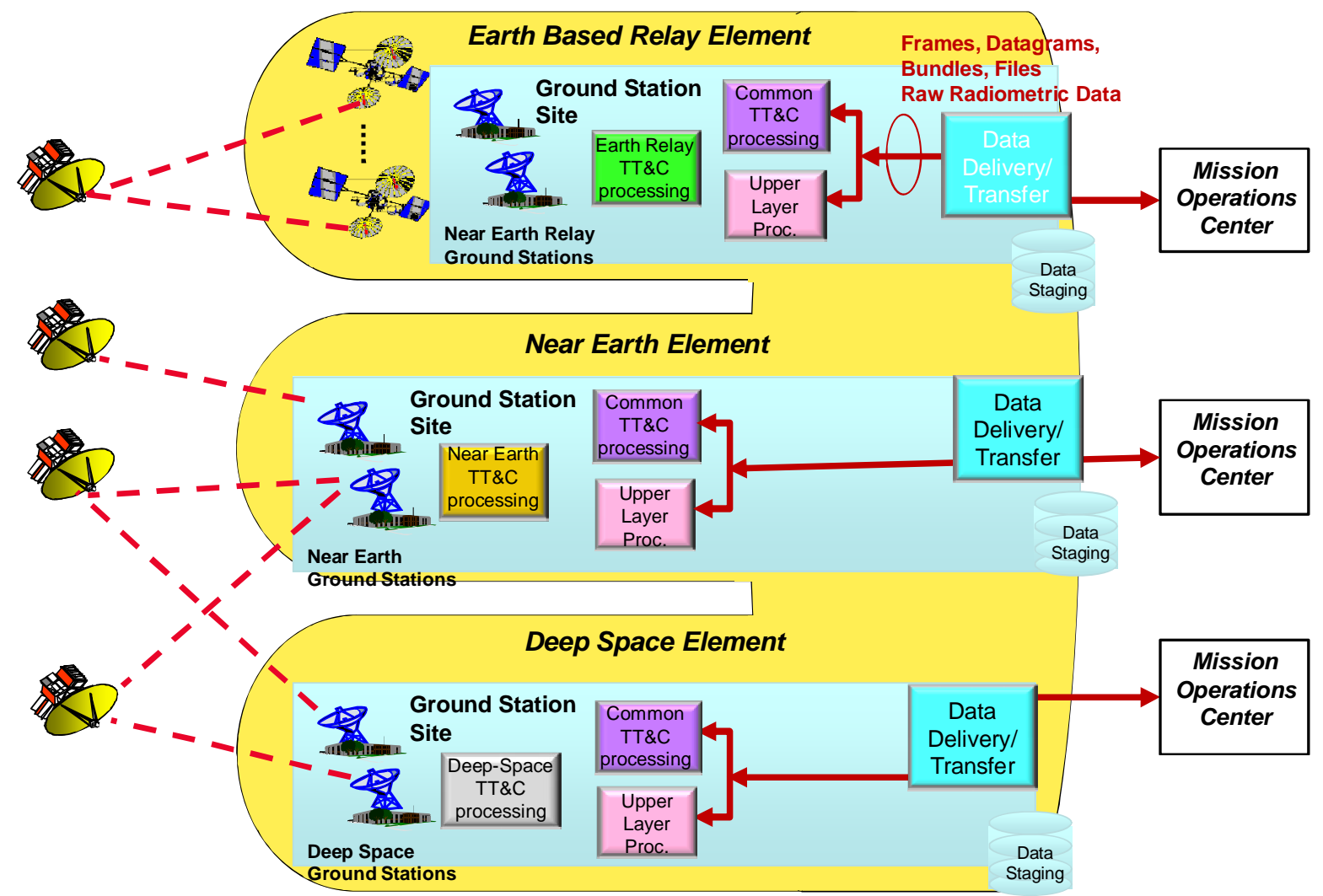

Figure 2. Integrated Network Architecture - Integrated Service Execution Function

The integrated service execution architecture has the following key attributes: 
- Standard services and service interfaces provided at all 3 network elements.

- Common (but distributed) service execution capabilities for all 3 network elements. High-degree of commonality in service execution equipment at all ground station sites (GSS) for all network elements.

- Common service execution capabilities based on EBRE developed capabilities (hardware \& software) with network asset-specific adaptation.

In addition, the architecture is adjusted to incorporate an important feature of the physically centralized options. A system element, the Service Portal is added to function as the virtually centralized data delivery point, i.e., the "service dispatcher", to user missions.

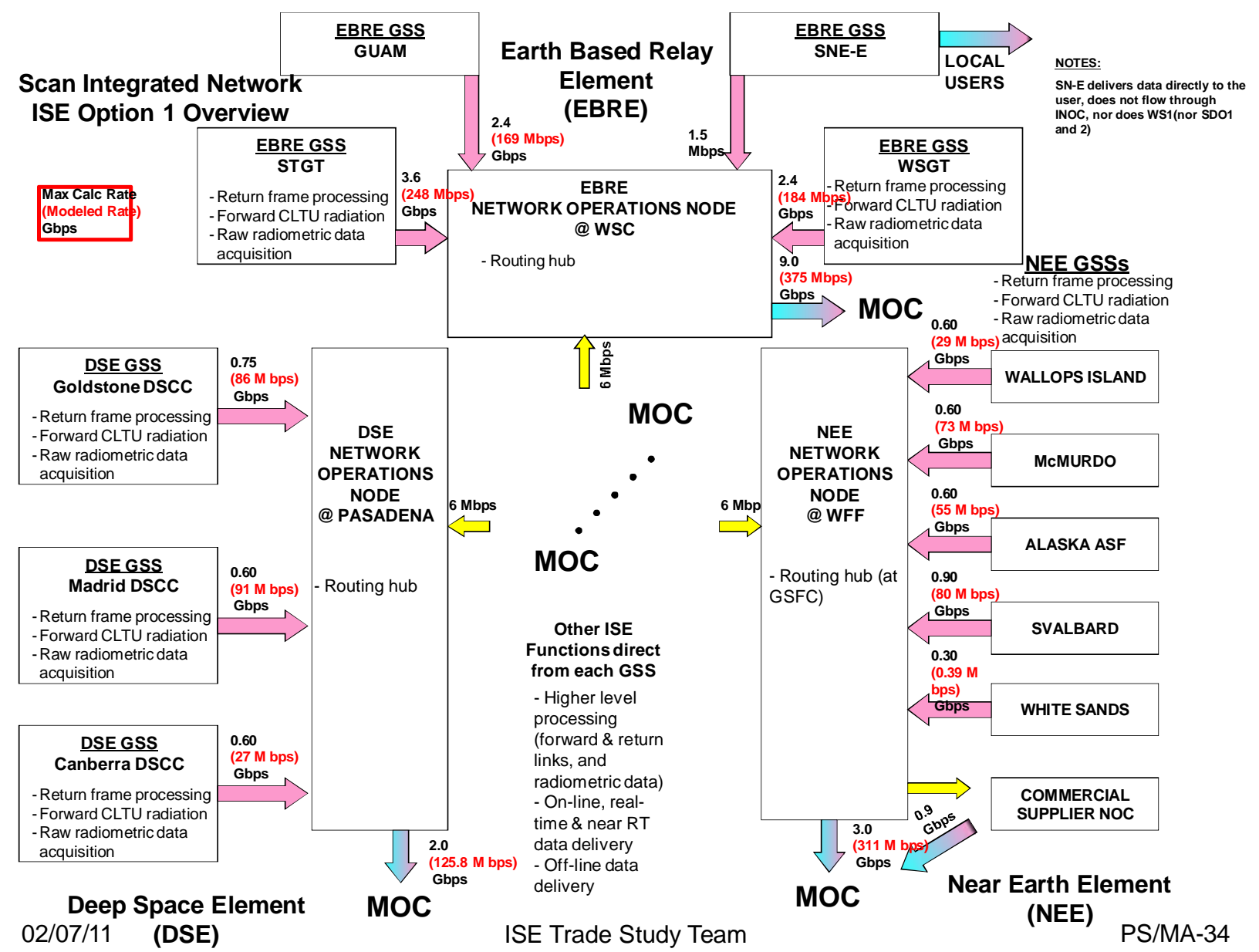

Figure 3. Physical Topology of Integrated Network Architecture for Service Execution Function

\section{B. Integrated Service Management}

Service Management consists of three functions: service planning, service request scheduling, and service accountability and reporting. These functions are typically performed in a sequential manner with only small amounts of overlap. The major features of each function are described below.

- Service Planning includes:

- Perform radio frequency (RF) link analyses and assess impacts

- Negotiate service agreements

- Plan and allocate resources based on tracking requirements; perform coverage analysis

- Perform loading analyses and assess impacts

- $\quad$ Service Request Scheduling includes:

- Negotiate contention resolution via peer-to-peer and/or priority based rule sets

- Develop top-level service schedules

- Service Accountability and Reporting includes: 
- Assemble, filter, and mine the relevant source data

- Assess service quality per service instance, missions, systems, networks and periodicity

- Generate service accountability reports for the integrated network

The future integrated network will provide mission users with a set of standard service management functions, primarily implemented using CCSDS service management standards. Interoperability between NASA network elements and assets operated by international partner agencies and commercial suppliers will be maximized.

The network will also maximize commonality in service management among the network elements. The network will dispatch mission user requests to individual network elements and coordinate among the network elements, thus reducing user burden, improving integration, and enabling an integrated service commitment process. A contextual view of the service management functions, showing relationships and high-level data flows is shown in Fig

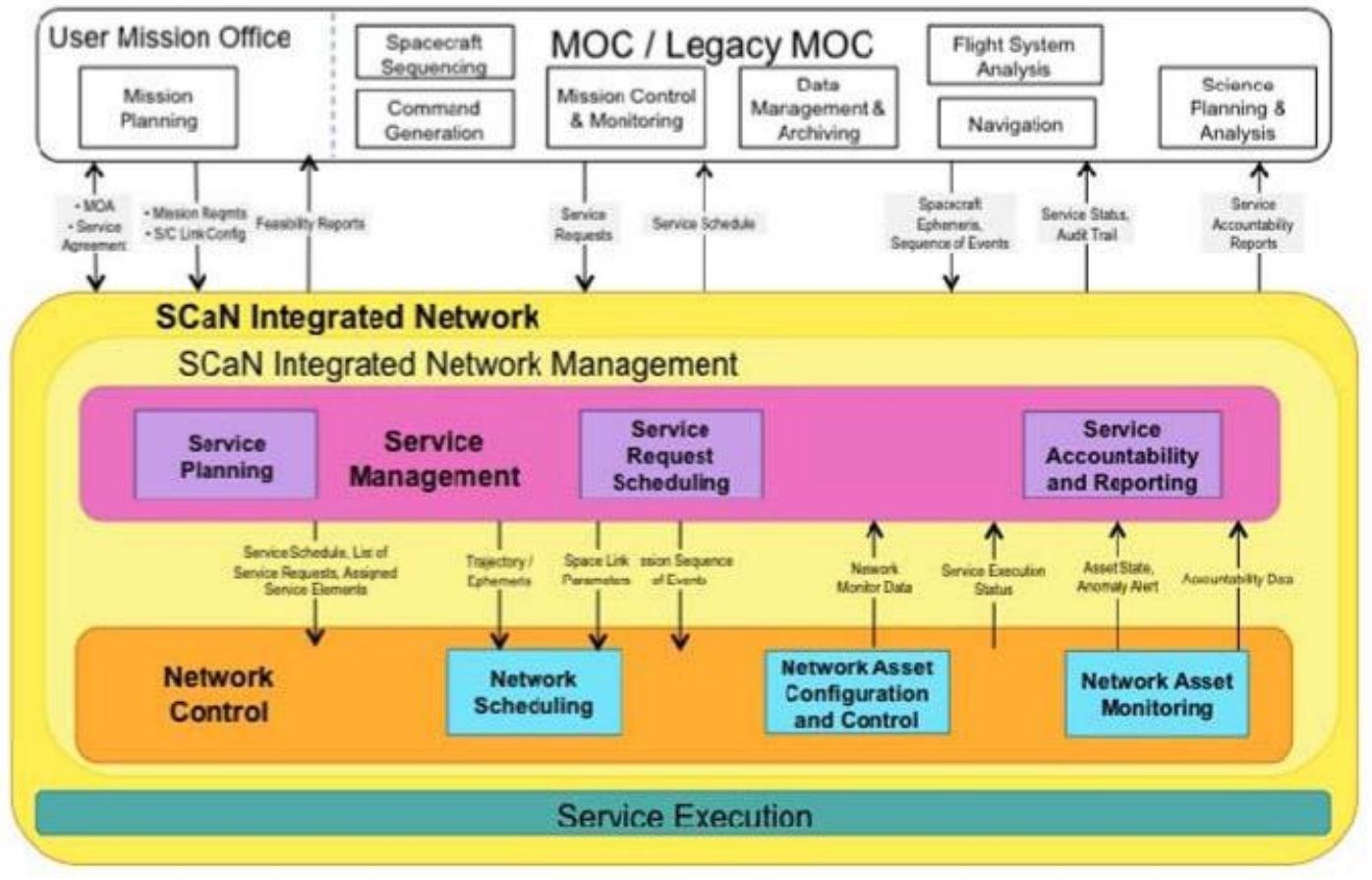

Figure 4. Integrated Network Architecture - Integrated Service Management Function

For the integrated service management architecture, two options were assessed: (1) The physically centralized service management performed at a single Integrated Network Operations Center (INOC); (2) The common (but distributed) service management capabilities performed at the Network Operations Node (NON) of each network element. The present network element-specific service management will cease to exist. Figure 5 shows the reference design for the centralized ISM architecture. The key characteristics of the centralized ISM option are:

1) A single INOC is implemented to perform the centralized service management functions for all the network assets of the IN.

2) Coordination is performed within the INOC for support of users needing service from more than one asset class.

3) Centralized service management will need to incorporate geographic redundancy for COOP, thus adding to the architecture option cost.

4) Interfaces to asset specific network control will require network element specific gateway interfaces.

Figure 6 shows the reference design for the common, distributed ISM architecture. The key characteristics of the common ISM option are:

1) Common service management functions are implemented with standard service interfaces and behavior. There may be more than one implementation of these common service management functions.

2) The set of service management capabilities are multiply deployed and operated, i.e., one for each NONassociated with a site or group of sites.

3) Coordination is required among service management instances for support of users needing service from more than one NON.

4) Distributed service management and network control will provide geographic redundancy for COOP. 


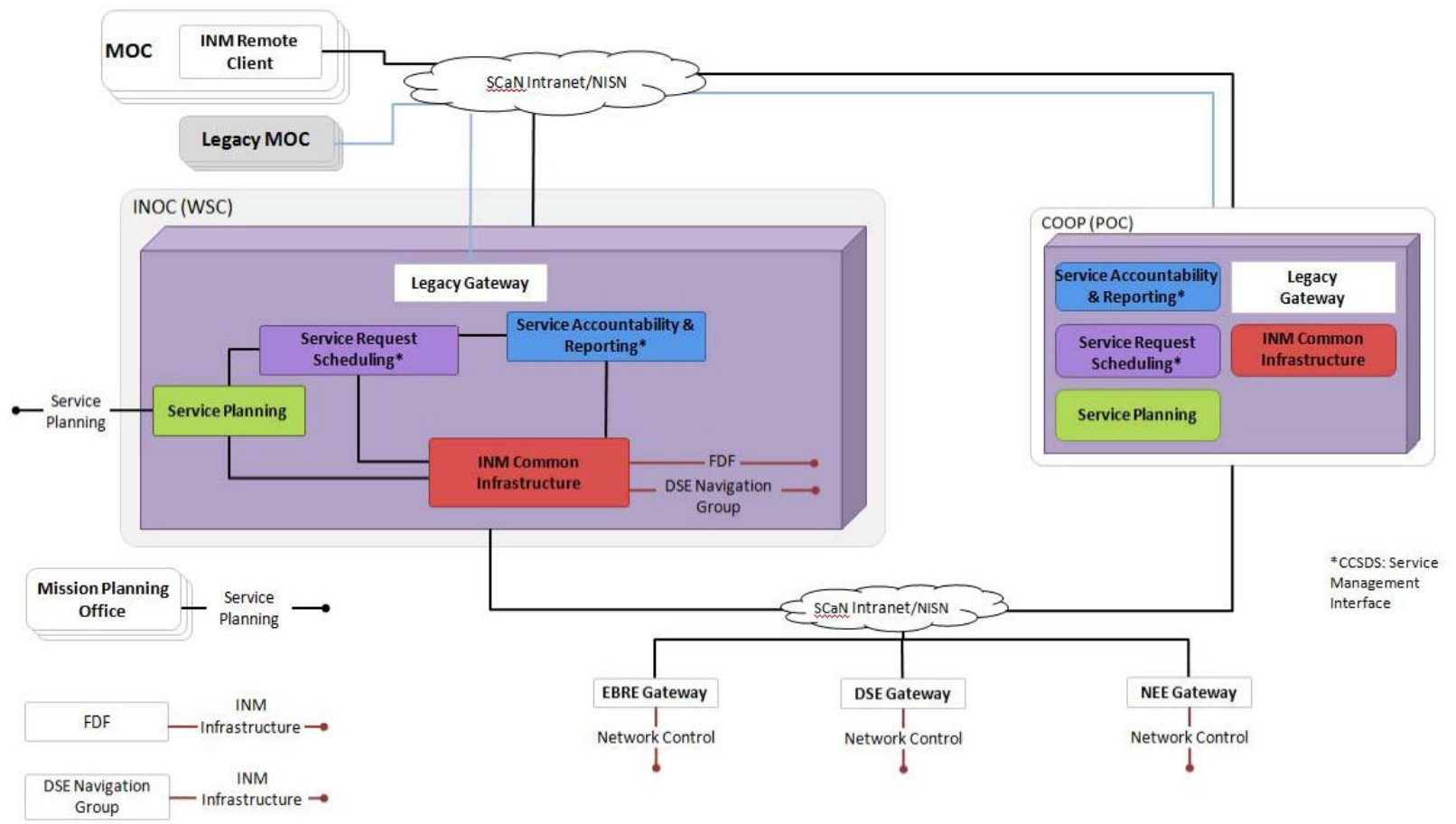

Figure 5. Centralized Service Management - Reference Design

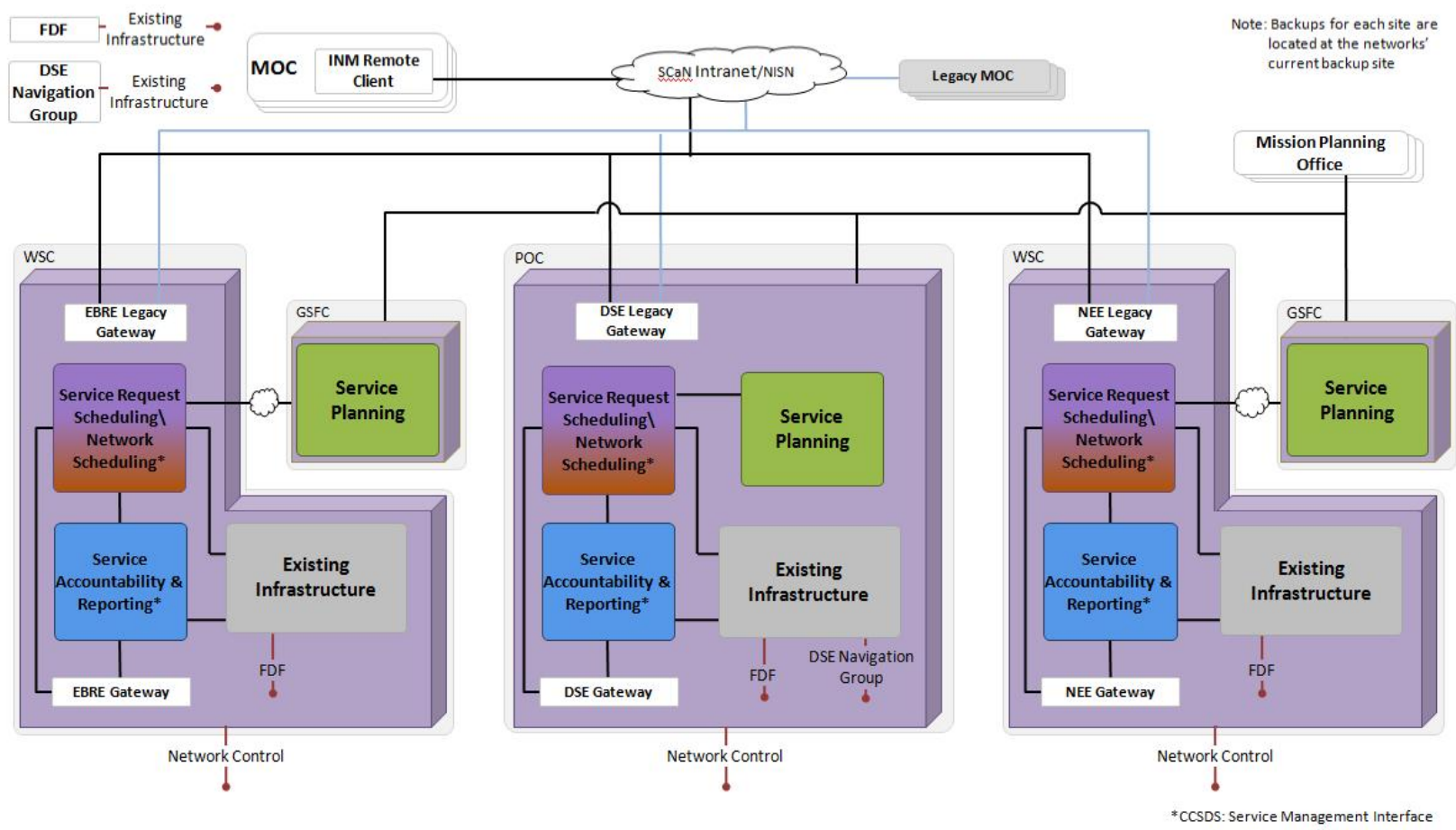

Figure 6. Common Service Management - Reference Design

Regardless of which of the two options is of better value, it is determined that, as the initial step, a Service Portal serving as the primary access point for user missions to interface with the NASA Integrated Network for service management and network control capabilities must be built. The Service Portal, from the view of user missions, is a web-based system facilitating the following service management activities: 
- Access to the integrated network services catalog, services catalog for each network element, and user's guide for each network element.

- Access to the MOU, MOA, service level agreements, IRDs, and ICDs.

- Interfaces with the Integrated Network for "service requests" in accordance with the CCSDS Service Management standard.

- Interfaces with the Integrated Network for submitting spacecraft trajectory files in accordance with the CCSDS Service Management standard.

- Interfaces with the Integrated Network for submitting spacecraft link configuration files in accordance with the CCSDS Service Management standard.

- Access to the all the network schedule products.

- Interfaces with the Integrated Network for viewing and receiving service accountability report at the end of each pass (i.e., for pass summary) and periodically (i.e., for daily, weekly, monthly, yearly, and mission-todate reports).

- Access to the RF link analysis tool(s) for performing the link design.

- Access to the results of coverage analysis performed by the integrated network.

- Access to the results of loading analysis performed by the integrated network.

- Access to the scheduling tool(s) for entering and editing scheduling input (and service request) parameters.

\section{Integrated Network Control}

The Network Control includes primarily (1) the network asset configuration and control and (2) network asset monitoring functions. The integrated network control architecture is addressed from two different aspects: the network control software and network control operations.

For assessing the integrated network control software (NCS) architecture, four options, as shown in the following table, were selected as the basis for the analysis of alternatives (AoA). Differentiating by the degree of the integrated nature and commonality, they range from the common network control framework to common interface software to gateway approaches.

\begin{tabular}{|l|l|l|}
\hline \multicolumn{2}{|c|}{ Software Alternatives } & \multicolumn{1}{c|}{ Description } \\
\hline $\begin{array}{l}\text { Option 1 } \\
\text { (NCS-1) }\end{array}$ & $\begin{array}{l}\text { Common network } \\
\text { control framework }\end{array}$ & $\begin{array}{l}\text { Common software framework for the entire network control functionalities } \\
\text { across all network elements, i.e., EBRE, NEE, and DSE. Such a software } \\
\text { framework includes common code providing generic network control } \\
\text { functionality, but can be selectively adapted or specialized by network } \\
\text { elements, thus accommodating network asset-specific functionality. }\end{array}$ \\
\hline $\begin{array}{l}\text { Option 2 } \\
\text { (NCS-2) }\end{array}$ & $\begin{array}{l}\text { Common network } \\
\text { control interface }\end{array}$ & $\begin{array}{l}\text { Common software components (within the network control function) that } \\
\text { provide the interfaces with human operators, service management, and user } \\
\text { mission elements. }\end{array}$ \\
\hline $\begin{array}{l}\text { Option 3 } \\
\text { (NCS-3) }\end{array}$ & Central gateway & $\begin{array}{l}\text { A singly implemented and centrally deployed gateway that functions as the } \\
\text { single interface point (for the network control function) with the service } \\
\text { management and user mission elements. The gateway performs necessary } \\
\text { protocol conversions for the dissimilar and network asset-specific network } \\
\text { control interfaces at the various network elements, i.e., EBRE, NEE, and } \\
\text { DSE. }\end{array}$ \\
\hline $\begin{array}{l}\text { Option 4 } \\
\text { (NCS-4) }\end{array}$ & $\begin{array}{l}\text { Network element } \\
\text { gateway }\end{array}$ & $\begin{array}{l}\text { Multiply deployed gateways that function as the interface points (for the } \\
\text { network control function) with the service management and user mission } \\
\text { elements through common interface protocols. The gateway at each network } \\
\text { element, i.e., EBRE, NEE, and DSE, performs necessary protocol } \\
\text { conversions for the dissimilar and network asset-specific network control } \\
\text { interfaces in each element. }\end{array}$ \\
\hline
\end{tabular}

Figure 7 depicts the system context for each integrated network control software architecture alternative. 
After evaluating all the options against a set of figures of merit (FOMs) and taking into account the risks, and cost estimates, the NCS-1 "Common Network Control Framework" option is selected. Key attributes of this option are as follows:

1) A common software framework, derived from the EBRE software, is the core for the network control software at all three network elements, EBRE, NEE, and DSE.

2) The common software framework will provide common, standard interfaces to:

- Mission Operations Centers (MOCs) of user missions

- Service Management elements resident at INOC (if Centralized Service Management is selected) or at NON and Service Portal (if Common Service Management is selected).

- Service Execution elements

- Network control operators

3) Specializations and adaptations of the common software framework for network element-specific network control capabilities will be primarily only through configuration files and scripts

4) The common software framework will be singly developed and sustained, but multiply deployed. The scripts and configuration files will be created and maintained locally at each network element.

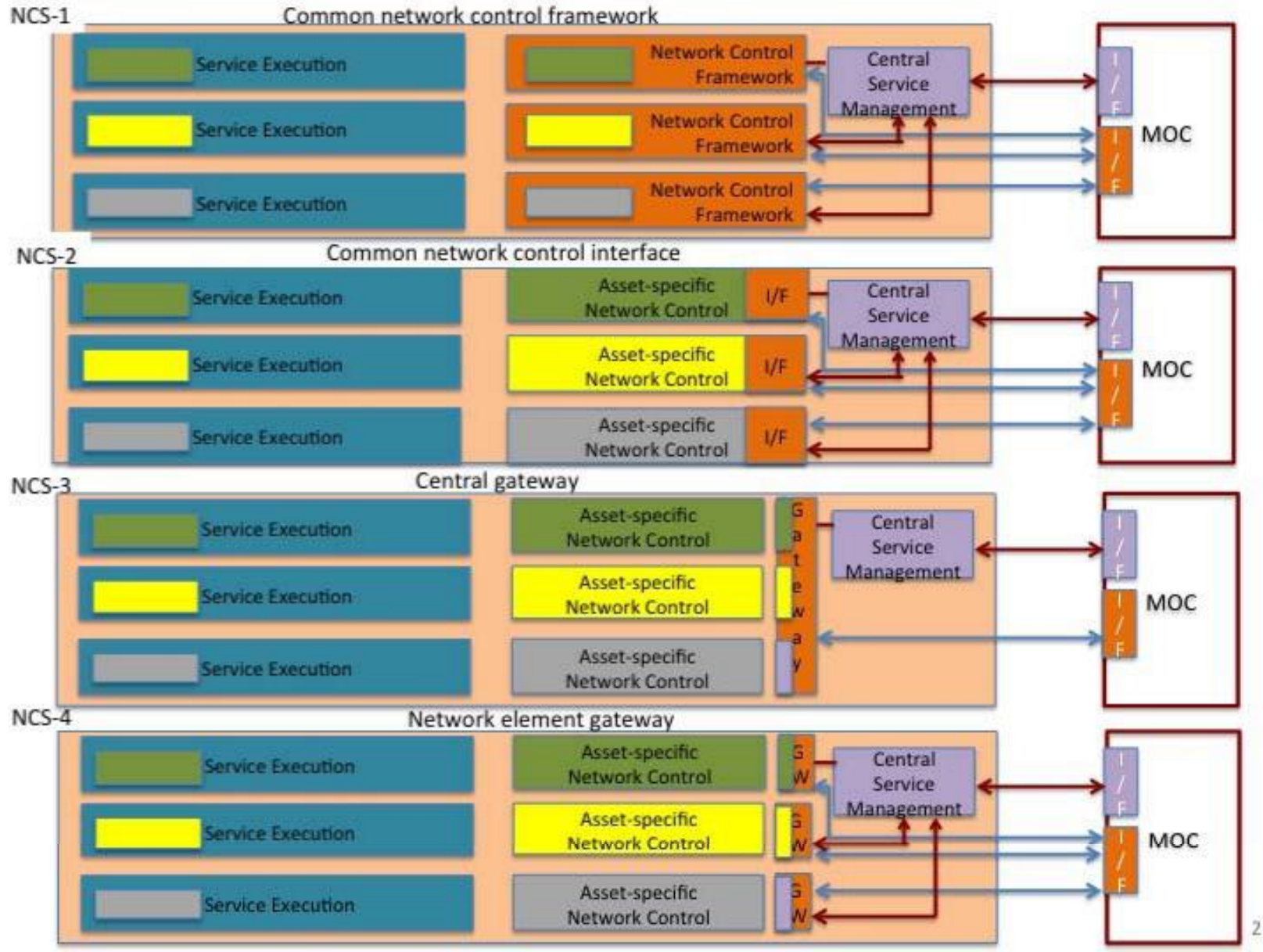

Figure 7. Integrated Network Control Software Architecture - System Context Per Options

For assessing the integrated network control operations (NCO) in terms of processes and operations team structure and deployment, three options, as shown in the following table, were selected as the basis for AoA. Differentiating by the degree of the integrated nature and commonality, they range from a fully integrated network control operations approach to one featuring operations cross support by demands. Moreover, as an inherent part of the NCO AoA, the back-up approaches to achieving continuity of operations (COOP) in events of disaster are factored into the overall trades. Three options, COO-1, -2 , and -3 , were identified. They are discussed in a separate paper presented at SpaceOps'2012. 


\begin{tabular}{|l|l|l|}
\hline \multicolumn{2}{|c|}{ Operations Alternatives } & \multicolumn{1}{c|}{ Description } \\
\hline $\begin{array}{l}\text { Option 1 } \\
\text { (NCO-1) }\end{array}$ & $\begin{array}{l}\text { Fully integrated } \\
\text { network control } \\
\text { operations }\end{array}$ & $\begin{array}{l}\text { The operational activities for network control are conducted globally at three } \\
\text { Network Operations Nodes (NONs) where each of the three NONs monitors } \\
\text { and controls the entire integrated network (EBRE, NEE and DSE) during a } \\
\text { single prime-shift. At each NON, operators for all network elements are } \\
\text { physically co-located. }\end{array}$ \\
\hline $\begin{array}{l}\text { Option 2 } \\
\text { (NCO-2) }\end{array}$ & $\begin{array}{l}\text { Partially integrated } \\
\text { network control } \\
\text { operations }\end{array}$ & $\begin{array}{l}\text { The operational activities for network control for each network element } \\
\text { (EBRE, NEE, or DSE) are conducted at each Network Operations Node } \\
\text { (NON). Cross-support among these NONs occurs for critical events, asset } \\
\text { contention period (ACP), COOP, and other events demanding additional } \\
\text { operational workforce for a network element. }\end{array}$ \\
\hline $\begin{array}{l}\text { Option 3 } \\
\text { (NCO-3) }\end{array}$ & $\begin{array}{l}\text { Asset-specific } \\
\text { network control } \\
\text { operations }\end{array}$ & $\begin{array}{l}\text { The operational activities for network control for each network element } \\
\text { (EBRE, NEE, or DSE) are conducted at each Network Operations Node } \\
\text { (NON). No cross-support among these NONs takes place except for COOP } \\
\text { purpose. }\end{array}$ \\
\hline
\end{tabular}

Figure 8 depicts the system context for each integrated network control operations alternative.

\section{NCO-1 Fully integrated network control operations}
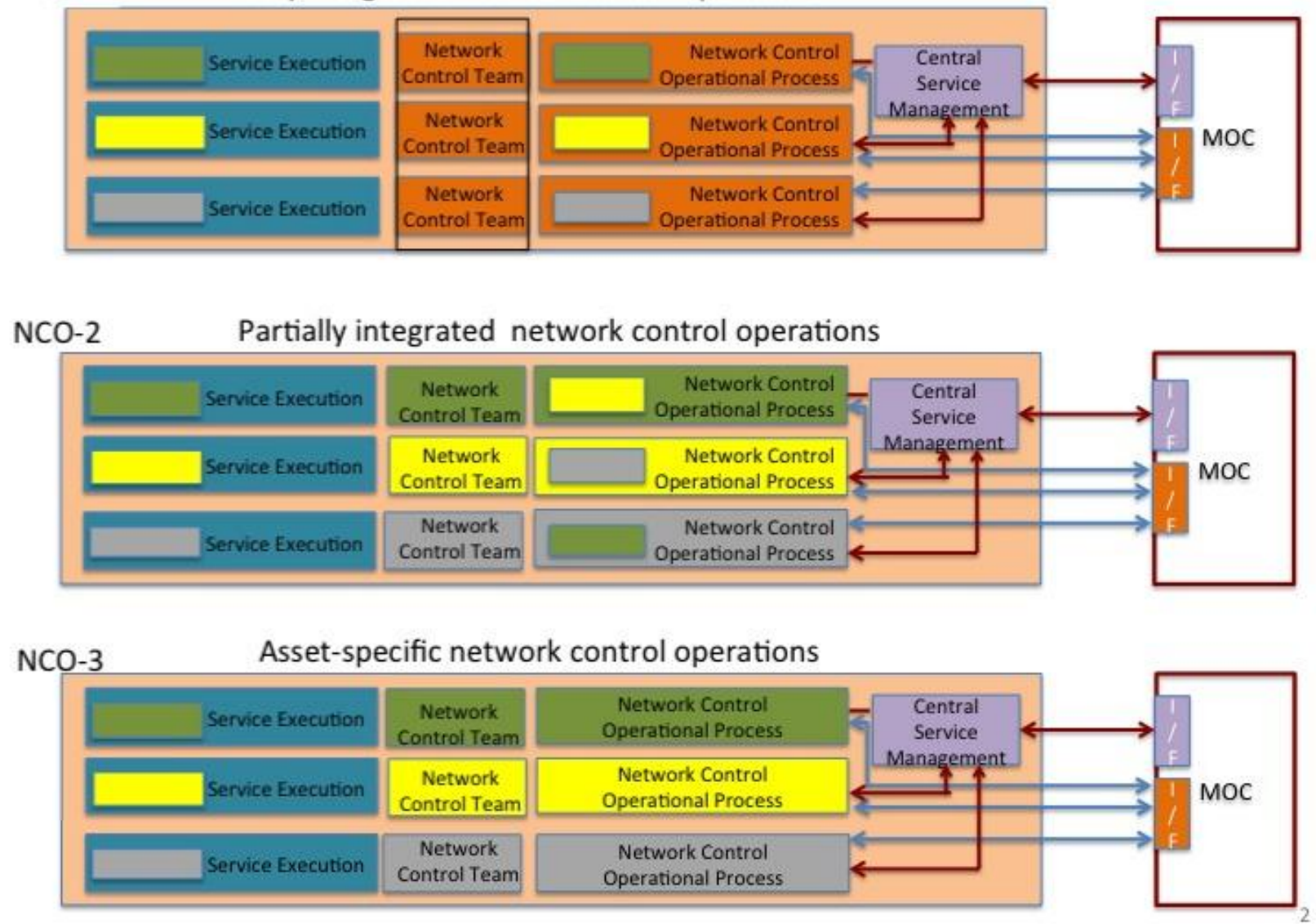

Figure 8. Integrated Network Control Operations Architecture - System Context Per Options

In NCO-1 option, termed "Fully Integrated Network Control Operations", the operational activities for network control are conducted globally at three Network Operations Nodes (NONs) where each of the three NONs monitors and controls the entire integrated network (EBRE, NEE and DSE) during a single prime-shift. Key attributes of NCO-1 are as follows: 
1) A single Network Operations Node (NON) is responsible for network monitoring and control functions at any given time.

2) The network control operations for the entire network, regardless of which network elements (EBRE, NEE, or DSE), are conducted at the prime-shift NON for a given shift (8 hours nominally). A follow-thesun approach.

3) At each NON, operators for all network elements are physically co-located.

4) Features a common, standard operational process for network control across all network elements (EBRE, NEE, and DSE).

5) Features a single integrated operational team, although its members are distributed physically at the various NONs, that conducts the network monitor and control activities.

6) A firewall may be established for the special-class mission support to insulate it from other missions support. Only the NON at the EBRE conducts the special-class missions support. In other words, the follow-the-sun rotation approach does not apply to special-class missions support.

7) This option can be best accommodated by the Common Network Control Framework option for the software.

8) Implies that all NONs must be equipped with network control software for all network elements.

9) Implies that the integrated operational team must be trained extensively for monitoring and controlling all network elements.

10) Implies that a high-degree of cross support among the NONs can be achieved when needed.

11) Implies that the optimization on operations efficiency can be further assessed by taking into account the operational and maintenance personnel at each NON or GSS locally, as well as across the NONs.

With the goal of achieving the fully integrated state for network control, via the NCS-1/NCO-1 solution, an evolution path should be taken:

1) Proceed with NCS-1 prototype to evaluate functionality and viability of the common network control framework.

2) Proceed with NCO-2, the partially integrated network control operations, using the NCS-1 prototypes to assess viability of integrated operations according to NCO-2 or NCO-1.

3) If NCS-1 is deemed too hard to achieve due to cost and/or other circumstances, fallback to NCS-3, asset specific network control systems, retain common ISE, service management, \& user missions interfaces.

4) If NCO-2 (or NCO-1) evaluations fail fallback to NCO-3 resulting in NCS-3/NCO-3 solution.

5) If NCO-2 (or NCO-1) evaluations prove their merits, move forward to the fully integrated state with the NCS-1 deployment and NCO-1 operations.

Moreover, it is determined that, as the initial step, a Service Portal serving as the primary access point for user missions to interface with the NASA Integrated Network for service management and network control capabilities must be built. The Service Portal, from the view of user missions, is a web-based system facilitating the following network control activities:

- Interfaces with the Integrated Network for viewing and receiving service execution status, alarm/alert, and monitor data during the pass and post-pass in compliance with the CCSDS CSTS monitor data standard.

- Interfaces with the Integrated Network for generating and submitting control directives for the setup of user mission-controllable configuration parameters during the pass and pre-pass in compliance with the CCSDS CSTS service control standard.

- Access to the SLE configuration parameters, i.e., those for establishing SLE "service binding" between the user mission's MOC and a service providing station of the Integrated Network.

\section{End State Architecture}

Given the conclusions for each of the three functions as stated in Section IV, the overall architecture for the NASA integrated space communications network has emerged. Figure 9 illustrates the end state architecture. The three network elements will have common service execution and network control at the various ground station sites (GSSs). The three network operations nodes (NONs) will have common network control capabilities as well to conduct the global, remote network monitor and control operations using the follow-the-sun approach. A service portal will provide human-machine interface to user missions for their service management and network control activities. The portal will eventually become an integral part of the integrated network operations center (INOC) that 
facilitates the centralized service management functions as the various network elements migrate out of the common service management paradigm.

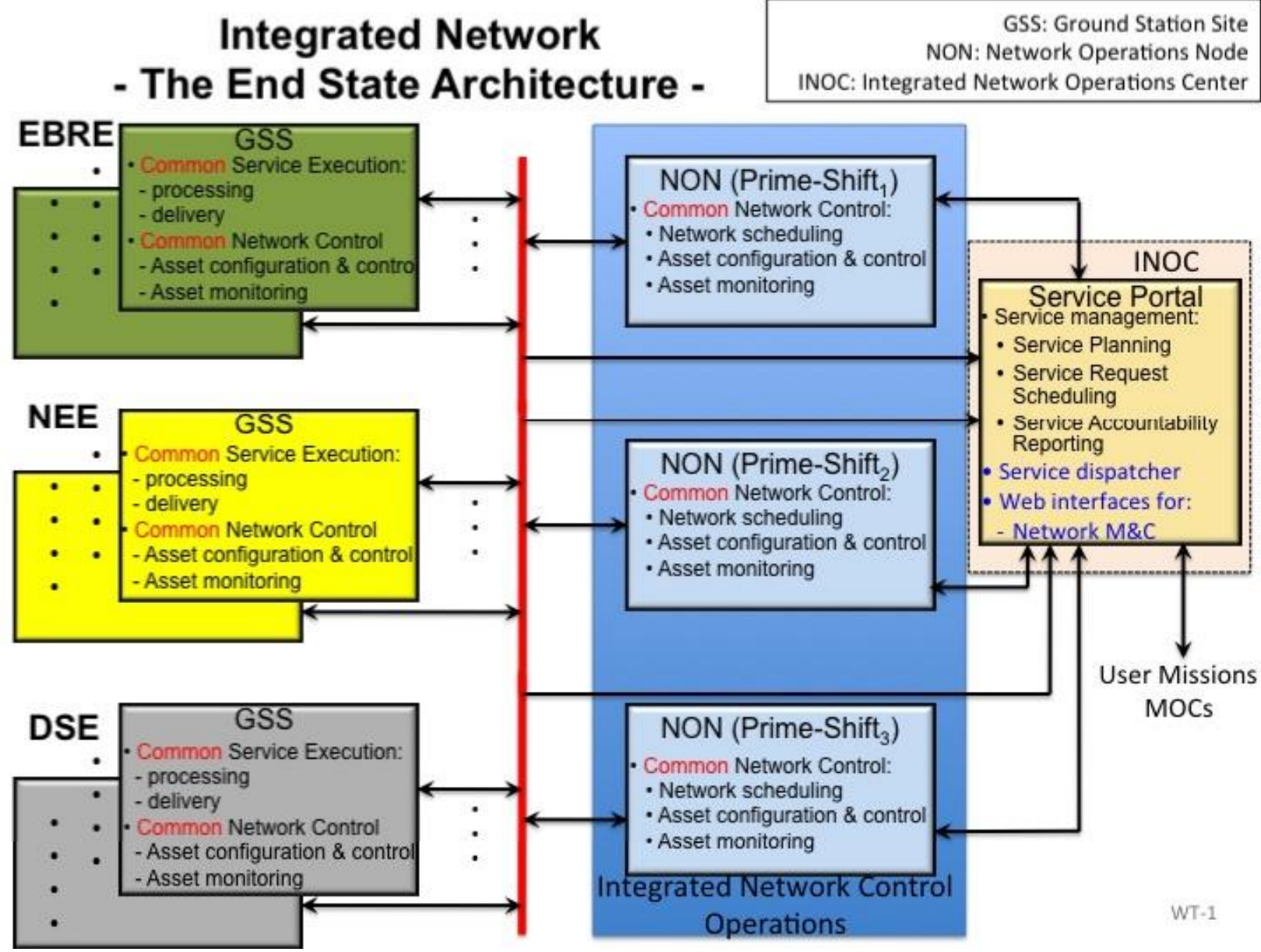

Figure 9. Integrated Network - The End State Architecture

\section{Conclusions}

The integrated space communications network defined so far has provided a foundation for programmatic planning at NASA. An incremental, phased development approach to realizing the integrated network is being formulated. Moreover, NASA will proceed with the implementation of certain pieces of the integrated network as the initial effort toward the end state architecture. 


\section{Appendix A \\ Acronym List}

\begin{tabular}{ll} 
ACP & Asset Contention Period \\
AoA & Analysis of Alternatives \\
CCSDS & Consultative Committee for Space Data Systems \\
COO & Continuity of Operations Option \\
COOP & Continuity of Operations \\
CSTS & Cross Support Transfer Service \\
DSE & Deep Space Element \\
DSN & Deep Space Network \\
EBRE & Earth Based Relay Element \\
EI & Enterprise Infrastructure \\
FOM & Figure of Merit \\
GEO & Geostationary Earth Orbit \\
GSS & Ground Station Site \\
GT & Ground Terminal \\
HEO & High Earth Orbit \\
INC & Integrated Network Control \\
INOC & Integrated Network Operations Center \\
ISM & Integrated Service Management \\
ISE & Integrated Service Execution \\
LEO & Low Earth Orbit \\
MOC & Mission Operations Center \\
NCS & Network Control Software \\
NCO & Network Control Operations \\
NEE & Near Earth Element \\
NEN & Near Earth Network \\
NON & Network Operations Node \\
SCaN & Space Communication and Navigation \\
SLE & Space Link Extension \\
SN & Space Network \\
TDRSS & Tracking and Data Relay Satellite System \\
TT\&C & Telemetry, Tracking and Command \\
\hline &
\end{tabular}

\section{Acknowledgments}

The authors would like to acknowledge the high-quality work contributed by all other members of the NASA architecture trade study team: from GSFC, Mike Anderson and Steve Kowal; from JPL, Peter Shames, Mike Leveque, Kar-Ming Cheung, Doug Abraham, and Bruce MacNeal; from GRC, Richard Kunath, Jessica Reinert, and Patrick Barnes. Also we would like to express our gratitude to the NASA SCaN Program Office for chartering and funding the architecture trade study. The strong and dedicated management support by Jim Schier is appreciated.

\section{References}

${ }^{1}$ Liebrecht, P., Schier, J., Bhasin, K., Bibyk, I., Butler, M., Hudiburg, J., Tai, W., Shames, P., "NASA’s Space Communications Integrated Architecture," Proceedings of SpaceOps 2010 Conference, AIAA, 25-30 April 2010, Huntsville, Alabama.

${ }^{2}$ Tai, W., Wright, N., "Integrated Network Management (INM) and Integrated Service Execution (ISE); Analysis Cycle 1 Study Report 1” NASA SCaN-STUDY-INM-ISE, 12 August 2011. 